\title{
Ageratina neblinensis (Asteraceae, Eupatorieae), una nueva especie para el norte del Perú
}

\section{Ageratina neblinensis (Asteraceae, Eupatorieae), a new species from northern Peru}

\author{
Juan Montoya Quino1, Eliana linares Perea², Iván Torres Marquina³, José Alfredo Vicente \\ Orellana ${ }^{4}$ \& Antonio Galán de Mera**
}

\begin{abstract}
${ }^{1}$ Herbario CPUN, Departamento de Biología, Universidad Nacional de Cajamarca, Cajamarca, Perú. ${ }^{2}$ Estudios Fitogeográficos del Perú, Sánchez Cerro 219, Manuel Prado, Paucarpata, Arequipa, Perú.

${ }^{3}$ Laboratorio de Botánica, Facultad de Ciencias de la Salud (Farmacia), Universidad Privada Antonio Guillermo Urrelo (UPAGU), Jr. José Sabogal 913, Cajamarca, Perú.

${ }^{4}$ Laboratorio de Botánica, Facultad de Farmacia, Universidad CEU San Pablo, apartado 67, 28660 Boadilla del Monte, Madrid, España.

*agalmer@hotmail.com
\end{abstract}

\begin{abstract}
As a result of field work on the vegetation of the northern Peruvian Andes, a new species of Ageratina Spach subgen. Andinia R.M. King \& H. Rob. is described for the montane rain forests of the Department of Cajamarca (Peru). Description, illustrations and a key to separate the nor-Peruvian Andinia species, studied in different herbaria, are presented.
\end{abstract}

Ageratina Spach (Asteraceae, Eupatorieae), género con ca. 250 especies ampliamente distribuido desde los Estados Unidos hasta los Andes de Bolivia y Argentina (Robinson \& Holmes 2007), se caracteriza por presentar inflorescencias corimbiformes, capítulos con el receptáculo convexo, glabro o con escasos pelos diminutos, flósculos campanulados con corola blanca, violeta o púrpura, y aquenios prismáticos o subfusiformes, generalmente con 5 costillas longitudinales setulíferas o con glándulas, o con ambos. Se subdivide en 5 subgéneros: Ageratina, Andinia R.M. King \& H. Rob., Apoda R.M. King \& H. Rob., Klattiella R.M. King \& H. Rob. y Neogreenella R.M. King \& H. Rob. El subgénero Andinia agrupa árboles y arbustos de hojas coriáceas generalmente aserradas, flores con lóbulos papilosos por la parte interna y aquenios con glándulas o setas sobre las costillas. Se distribuye en los bosques húmedos del norte del Perú, Ecuador, Colombia y Venezuela (King \& Robinson 1987, Robinson 2006). Para el Perú aún no hay un tratamiento específico sobre el género, aunque se conocen 40 especies (Brako \& Zarucchi 1993), cifra ya modificada por el descubrimiento de nuevos registros (Robinson 2006, 2011, Robinson \& Holmes 2007).

Durante las recolecciones para abordar la vegetación de los bosques húmedos del Perú herborizamos un arbusto cuyas características, consultadas con material de los herbarios CPUN, F, G, GH, MA, NY, S y US, difieren del resto de las especies del subgénero Andinia que crecen en el norte del Perú, en el entorno de la depresión de Huancabamba $-5^{\circ} 47^{\prime} 24,08^{\prime \prime} \mathrm{S}$ / 79²6'33,44"'W - 5'50'13,89's / 78\%45'03,70'W (ver Weigend 2002)- (A. boekei R.M. King \& H. Rob., A. chachapoyasensis (Cuatrec.) R.M. King \& H. Rob., A. dillonii H. Rob., A. fastigiata (Kunth) R.M. King \& H. Rob. (= A. exserto-venosa (Klatt) R. M. King \& H. Rob.), A. lopez-mirandae R.M. King \& H. Rob., A. maranonii H. Rob., A. piurae (B. Rob.) R.M. King \& H. Rob. y A. wurdackii R.M. King \& H. Rob.), por sus hojas de ovadas a cordadas, sésiles o subsésiles, y flores con corola, anteras y ramas estilares violeta pálido.

Ageratina neblinensis J. Montoya, E. Linares, I. Torres, Vicente Orell. \& A. Galán, sp. nov.

TIPO: Perú, Departamento de Cajamarca, Cutervo, 6017'39,92"S-7849'19,65"W, 2.660 m, 5-VIII-2016, J. Montoya, E. Linares \& A. Galán 4608 (Holotipo CPUN!, isotipo USP!).

Shrubs up to $1.5 \mathrm{~m}$. Leaves, decussate, with petioles extremely short or without petioles, and blades 2-2.2 $x$ 1.3-1.8 cm, generally ovate to cordate, and serrate. Inflorescences corymbose with 3-5 heads 1.2-1.4 x 1.3-1.8 $\mathrm{cm}$ at anthesis, accompanied by an extra-head bract. Flower corollas pale violet, glabrous outside with lobes covered with papillae inside, style branches pale violet, papillose, erect to spreading. Achenes with short bristles on the ribs. 
Arbustos hasta de $1,5 \mathrm{~m}$ de altura, con tallos cilíndricos, tomentosos, pelos tectores y glandulíferos hasta de $0,5 \mathrm{~mm}$. Hojas decusadas, sésiles o subsésiles, con láminas de 2-2,2 x $1,3-1,8 \mathrm{~cm}$, generalmente de ovadas a cordadas, las pequeñas deltoides, aserradas, con nerviación reticulada, villosas por el envés y glabrescentes por el haz, con pelos tectores y algunos glandulíferos. Inflorescencias oligocéfalas, corimbiformes, formadas por 3-5 capítulos. Capítulos de 1,2-1,4 x 1,3-1,8 $\mathrm{cm}$ en la antesis, con ca. 70 flores; receptáculo ca. 2,5 x 2,5 $\mathrm{mm}$, convexo, escrobiculado, con pelos diminutos dispersos; involucro formado por ca. 20 brácteas en 3 series, las internas de 5,6-7,1 x 0,7-1,2 mm, las intermedias de 5-5,6 x 1,3-2,2 $\mathrm{mm}$, y las externas de 4,8-5,4 x 2,6-3,5 mm, lanceoladas, margen escarioso inconspicuo de ca. $0,2 \mathrm{~mm}$, con pelos tectores y glandulíferos, y 3-4 brácteas extracapitulares de $5,2-6,1 \times 2,2-2,8 \mathrm{~mm}$, semejantes a las más externas del involucro, la superior situada ca. 1-2 mm por debajo del capítulo. Flores campanuladas, hermafroditas; corola 4,3$5,5 \mathrm{~mm}$, violeta pálido, glabra por la parte externa, tubo de 4,1-4,8 mm y lóbulos de $0,5-0,8 \mathrm{~mm}$, papilosos por la parte interna; anteras de 1,7-2 mm, violeta pálido, polen de ca. 22$23 \mu \mathrm{m}$ de diámetro, equinulado, regular; ramas estilares de 3,3-4,1 x 0,2-0,6 mm, violeta pálido, papilosas, de erectas a patentes en la flor madura. Aquenios 2,4-3,2 x 0,6-0,7 mm, con algunas setas cortas sobre las costillas, concentradas en la mitad superior, sin glándulas, carpopodio de 0,1-0,2 mm. Vilano uniseriado, cerdas escábridas, de 3,6 a $5 \mathrm{~mm}$, blancas (Figs. 1 y 2).

Etimología: El epíteto específico se refiere a los "bosques de neblina" (bosques húmedos montanos de los Andes orientales) donde habita esta especie.
ObSERVACIONES: A. neblinensis vive en los bosques húmedos de los Andes orientales del norte peruano asociada con Alnus acuminata Kunth, Aulonemia longiaristata L.G.Clark \& Londoño, Chusquea scandens Kunth, Clethra revoluta (Ruiz \& Pav.) Spreng., Ctenitis sloanei (Poepp. ex Spreng.) C.V. Morton, Hedyosmum sprucei Solms, Monactis flaverioides Kunth, Monochaetum lineatum (D. Don) Naudin, Pteris quadriaurita Retz., Schefflera acuminata (Pav.) Harms y Weinmannia elliptica Kunth.

Las especies más relacionadas con A. neblinensis son A. chachapoyasensis y A. wurdackii, conocidas también del norte del Perú (Brako \& Zarucchi 1993). Con A. chachapoyasensis comparte corimbos con pocos capítulos, láminas de las hojas de ovadas a cordadas y los aquenios con algunas setas sobre las costillas; en cambio, con $A$. wurdackii, corimbos con pocos capítulos y hojas sésiles. $A$. chachapoyasensis destaca por sus flores con corola púrpura y hojas con largos pecíolos, mientras que $A$. wurdackii por corolas blancas y hojas con lámina elíptica. Aunque A. cutervensis (Hieron.) R.M. King \& H. Rob. pertenece al subgénero Ageratina, sus hojas coriáceas, con láminas de ovadas a deltoides y subsésiles, se asemejan a las de A. neblinensis, del subgénero Andinia. El hecho de que $A$. cutervensis muestre semejanzas con especies del subgénero Andinia ya fue resaltado por Robinson (1926) al describir Eupatorium piurae (A. piurae), aunque ésta es muy diferente a A. neblinensis por presentar hojas claramente pecioladas, inflorescencias policéfalas, densas, y flores con corola blanca.

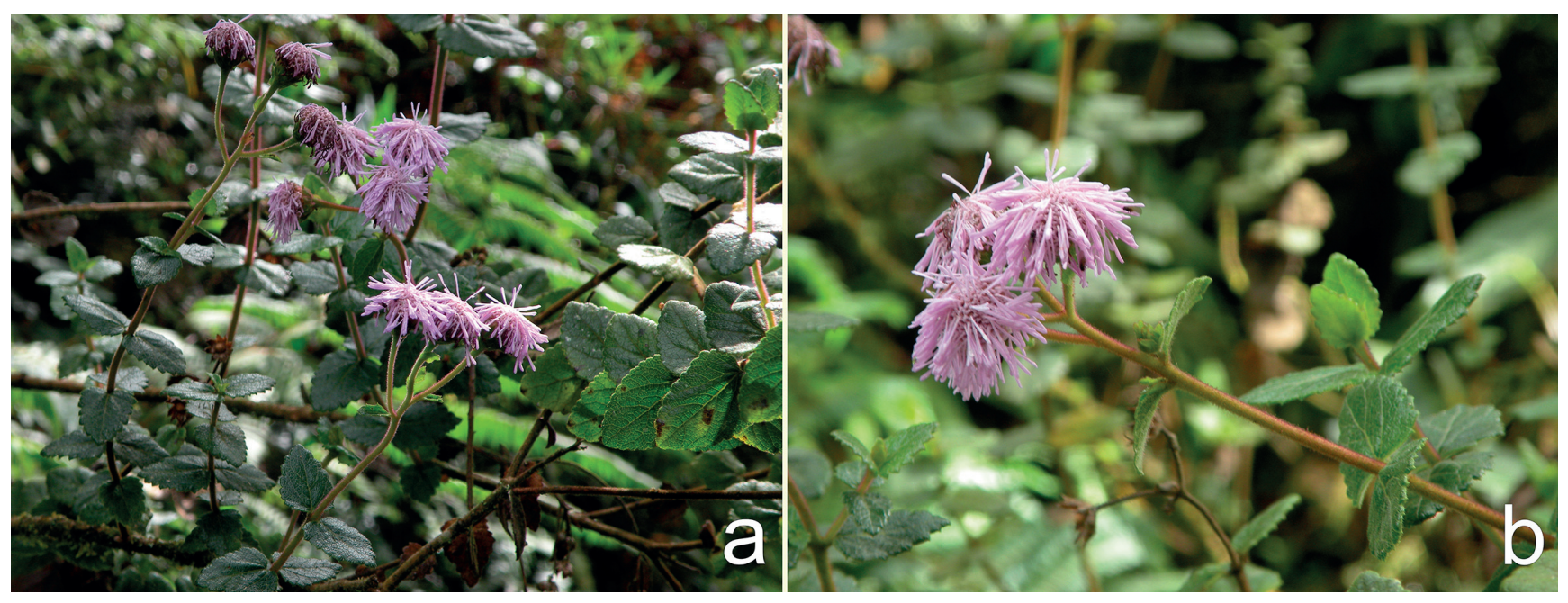

Figura 1. Ageratina neblinensis. a. Ramas con las inflorescencias; b. Detalle de una inflorescencia con las ramas estilares patentes en las flores. Fotos: Eliana Linares Perea. / Ageratina neblinensis. a. Branches with several inflorescences; b. Detail of an inflorescence showing the spreading style branches in its flowers. Photographs: Eliana Linares Perea. 

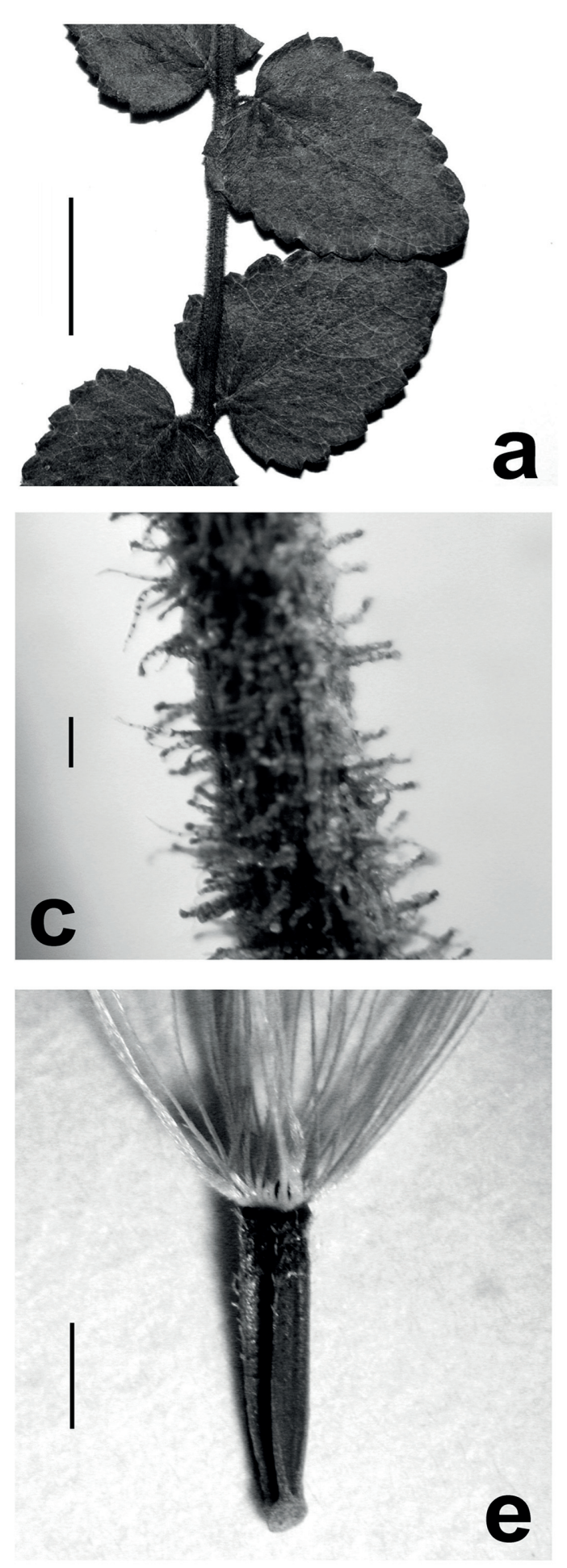
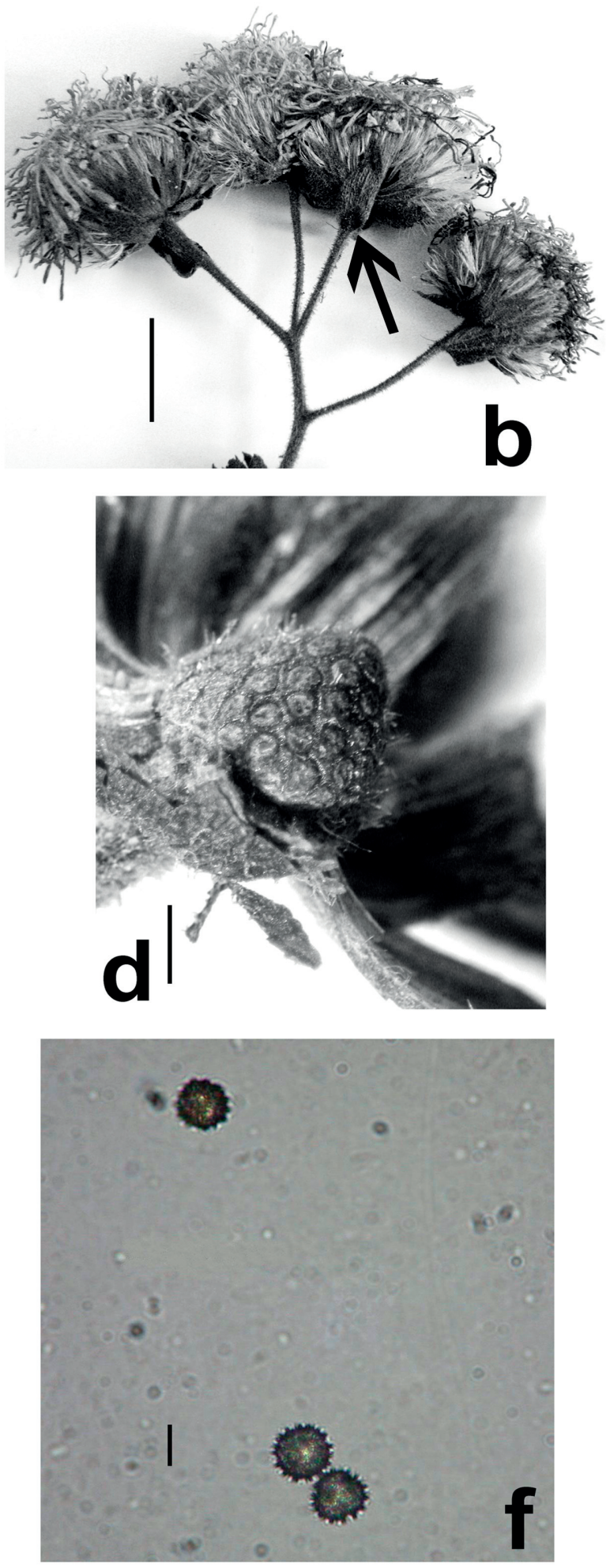

FiguRA 2. Ageratina neblinensis. a. Detalle de las hojas (barra $=1 \mathrm{~cm})$; b. Capítulos mostrando la bráctea extracapitular superior (barra $=1 \mathrm{~cm}$ ); c. Detalle del indumento del tallo (barra $=0,5 \mathrm{~mm}$ ); d. Receptáculo de un capítulo (barra $=1 \mathrm{~mm}$ ); e. Aquenio con parte del vilano (barra $=1 \mathrm{~mm}$ ); f. Granos de polen (barra $=20 \mu \mathrm{m}$ ). Fotos: a, b. Juan Montoya Quino. c, d, e, f. Antonio Galán de Mera. / Ageratina neblinensis. a. Detail of leaves ( $\mathrm{bar}=1 \mathrm{~cm}) ; \mathrm{b}$. Heads showing their extra-head highest bract $(\mathrm{bar}=1 \mathrm{~cm}) ; \mathrm{c}$. Detail of the steam indumentum $(\mathrm{bar}=0.5 \mathrm{~mm})$; . Receptacle of a head $(\mathrm{bar}=1 \mathrm{~mm})$; e. Achene with part of its pappus $(\mathrm{bar}=1 \mathrm{~mm}) ; \mathrm{f}$. Pollen grains $(\mathrm{bar}=$ $20 \mu \mathrm{m})$. Photographs: a, b. Juan Montoya Quino. c, d, e, f. Antonio Galán de Mera. 
Clave de Ageratina (Andinia) del norte del Perú:

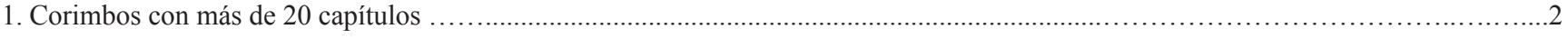

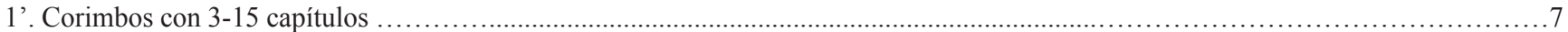

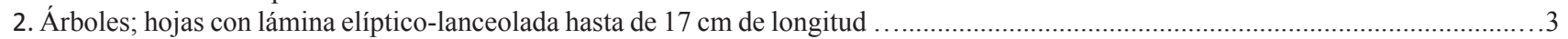

2'.Arbustos; hojas con lámina de ovada a cordada o elíptica hasta de $6,5 \mathrm{~cm}$ de longitud ...........................................

3. Corimbos con ca. 20-33 capítulos ca. 0,8 x 0,5 cm, con 12-15 flores; vilanos blancos; hojas con pecíolo 0,5-1 cm, lámina entera, a

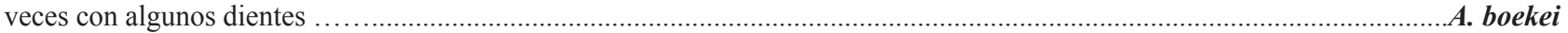
3'. Corimbos con ca. 80-90 capítulos ca. 0,7-0,9 x 0,4 cm, con 5-8 flores; vilanos rosados hacia la base; hojas con pecíolo 1-2 cm,

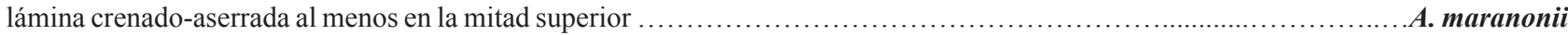
4. Tallos pubérulos; hojas con pecíolo 6-16 mm, lámina 1,8-6,5 x 1,5-3 cm, de elíptica a ovada, glabrescente por el envés, crenadoaserrada en la mitad superior

A. fastigiata 4'. Tallos tomentosos; hojas con pecíolo hasta de $9 \mathrm{~mm}$, lámina hasta de 5 x $2 \mathrm{~cm}$, de elíptica a ovada o elíptica, tomentoso-lanosa por el envés, crenado-aserrada o serrulada, a veces casi toda la lámina

5. Capítulos con ca. 15 flores; aquenios ca. $2,8 \mathrm{~mm}$, con glándulas sobre las costillas; hojas con lámina aserrada excepto en la base .. A. piurae

5'. Capítulos con ca. 10 flores; aquenios ca. $3,3 \mathrm{~mm}$, con glándulas y setas sobre las costillas; hojas con lámina crenado-aserrada o serrulada en la mitad superior....

6. Hojas con pecíolo 7-9 mm; lámina 2,5-3,5 x 1-2 cm, de elíptica a ovada, de apariencia entera aunque serrulada en la mitad superior

6'. Hojas con pecíolo 2-4 mm; lámina 2,5-5 x 0,7-2 cm, elíptica, crenado-aserrada en la mitad superior ..........A. lopez-mirandae

7. Corimbos con 3-5 capítulos 1,2-1,4 x 1,3-1,8 cm, con ca. 70 flores; corola violeta pálido ........................A. neblinensis

7'. Corimbos con más de 6 capítulos ca. 1 x $0,6-0,9 \mathrm{~cm}$, con $30-50$ flores, corola púrpura o blanca ........................... 8. Capítulos 0,9-1 x ca. 0,6 cm, con 35-50 flores, corola púrpura; hojas con pecíolo 10-20 mm, lámina 3-6 x 1,5-3,5 cm, de ovada a cordada

8'. Capítulos 1,1-1,2 x 0,6-0,9 cm, con ca. 30 flores, corola blanca; hojas sésiles, lámina 2,5-5,5 x 1,2-2,5 cm, elíptica

A. wurdackii

Materiales estudiados: Ageratina boekei: PERÚ. Departamento de Amazonas, Prov. Chachapoyas, Leimebamba-Lajasbamba trail, 27-VI-1977, J.D. Boeke (US, holotipo). A. chachapoyasensis: PERÚ. Departamento de Amazonas, Prov. Chachapoyas, uppermost slopes and summit of Cerros de Calla-Calla, near kms. 403-407 of Balsas-Leimebamba road, 3400-3550 m, 18-VIII-1962, J.J. Wurdack 1703 (US, holotipo). A. cutervensis: PERÚ. Sin localidad, V-1879, C. von Jelski 637 (F, holotipo). Sin localidad, H. Ruiz \& J.A. Pavón (MA). Departamento de Cajamarca, Prov. Celendín, Miguel Iglesias, 3526 m, 28-V2009, C. Tovar 724 (CPUN). Departamento de Cajamarca, Prov. Celendín, La Libertad de Pallán, 3513 m, 28-V-2009, C. Tovar (CPUN). A. dillonii: PERÚ. Departamento de Lambayeque, Prov. Ferreñafe, ca. 7 km NW of Incahuasi, near Cerro Punamachay on trail to Laguna Hualtaco, 3300-3550 m, 16-XI-1984, M.O. Dillon \& D. Skillman 4144 (US, holotipo). A. fastigiata: ECUADOR. Cantón de Loja, Prov. Loja, nudo de Sabanilla, W slope on road to Yangana, 6-II-1985, G.W. Harling \& B.L. Andersson (US). PERÚ. Departamento de Cajamarca, Prov. Cajamarca, entre Cerro Gavilán y Cajamarca, 3000 m, 25-V-1970, I. Sánchez Vega 579 (CPUN). Departamento de La Libertad, Prov. Huamachuco, borde de la Laguna Sausacocha, 3000 m, I. Sánchez Vega \& H. Bazán Zurita 1362 (CPUN). Departamento de La Libertad, Prov. Sánchez Cerro, Laguna
Sausacocha, between Huamachuco and Cajabamba, 18VIII-1982, D. Smith \& A. Cáceres (US). A. lopez-mirandae: PERÚ. Departamento de La Libertad, Prov. Otuzco, Cerro Sango, 3300-3400 m, 7-VI-1953, A. López M. 0972 (US, holotipo). A. maranonii: PERÚ. Departamento de Amazonas, Prov. Bongará, jalca zone $3 \mathrm{~km}$ south of Pomacocha, east of Shipasbamba trail, 2400 m, 20-VI-1962, J.J. Wurdack 975 (US, holotipo; NY, isotipo). A. piurae: PERÚ. Departamento de Piura, Prov. Huancabamba, Above Huancabamba, 3200-3300 m, IV-1912, A. Weberbauer (F, holotipo; GH isotipo). A. wurdackii: PERÚ. Departamento de Amazonas, Prov. Chachapoyas, Cerros Calla-Calla, east side, $19 \mathrm{~km}$ above Leimebamba on the road to Balsas, 3100 m, 7-VI-1964, P.C. Hutchinson \& J.K. Wright 5574 (US, holotipo; F, isotipo).

\section{AGRADECIMIENTOS}

Este trabajo ha sido realizado con los fondos del proyecto "Mapa de vegetación de Cajamarca. Potencialidad de la vegetación para el uso de plantas medicinales" de la Universidad Privada Antonio Guillermo Urrelo (Cajamarca, Perú), dentro del convenio de cooperación firmado con la Universidad CEU San Pablo (Madrid, España), y del proyecto-autorización (AUT-IFL-2017-011) del Servicio 
Nacional Forestal y de Fauna Silvestre (SERFOR) del Perú. Gracias a Harold Robinson y Hamilton Beltrán por la revisión crítica del primer manuscrito. A Julio Pinto por su ayuda logística durante los trabajos de campo y herbario.

\section{LITERATURA CITADA}

Brako, L., Zarucchi, J.L. 1993. Catálogo de las Angiospermas y Gimnospermas del Perú. Missouri Botanical Garden, St. Louis. 1286 pp.

King, R.M., Robinson, H. 1987. The genera of the Eupatorieae (Asteraceae). Monographs in Systematic Botany from the Missouri Botanical Garden, St. Louis. 581 pp.
RoBINSON, B.L. 1926. Records preliminary to a general treatment of the Eupatorieae VI. Contributions from the Gray Herbarium of Harvard University 77: 3-62.

Robinson, H. 2006. New species of Ageratina from Andean South America (Eupatorieae: Asteraceae). Phytologia 8(2): 154175.

Robinson, H. 2011. Ageratina tovarae, a new species from northern Peru (Asteraceae, Eupatorieae). Phytologia 93(1): 94-97.

Robinson, H., Holmes, W.C. 2007. Compositae-Eupatorieae. In: Harling, G., Persson, C. (eds.), Flora of Ecuador, Vol. 83 $(190,3)$, pp. 4-349. University of Göteborg, Stockholm.

Weigend, M. 2002. Observations on the Biogeography of the Amotape-Huancabamba Zone in Northern Peru. The Botanical Review 68(1): 38-54.

Recibido: 18.07.2017

Aceptado: 16.10.2017 